\title{
Conditions for Stabilization of the Tokamak Plasma Vertical Instability Using Only a Massless Plasma Analysis
}

\author{
J. William Helton \\ Kevin J. McGown \\ M. L. Walker
}

\begin{abstract}
This paper describes the problem of feedback control for stabilization of the plasma vertical instability in a tokamak. Such controllers are typically designed based on a model that assumes the plasma mass $m$ is identically zero while in reality the mass is small but positive. The assumption that $m$ is zero can lead to a controller that appears to be stabilizing according to the massless analysis but in fact can increase the instability of the physical system.

In this work, we consider a general class of controllers, which contains as a special case the type of controller most commonly used in operating tokamaks to stabilize the vertical instability, a proportional-derivative controller. Suppose $C$ is a controller in this class which stabilizes the vertical instability with plasma mass assumed to be zero. We give easy-to-check necessary and sufficient conditions for $C$ to also stabilize the physical system, in which the plasma actually has a small mass. We allow for the possibility that the tokamak could have both superconducting and resistive conductors.

The practical implications of the results presented provide substantial insight into some long-standing issues regarding feedback stabilization of the vertical instability with $P D$ controllers and also provide a rigorous foundation for the common practice of designing controllers assuming $m=0$. For controllers that operate only on the plasma vertical position, we settle the question: when are $m=0$ models predictive of actual plasma behavior?
\end{abstract}

\section{INTRODUCTION}

This paper considers the problem of feedback control for stabilization of the vertical instability in tokamaks. Tokamaks are torus-shaped devices designed to confine a plasma composed of ionized hydrogen isotopes while the plasma is heated to initiate fusion reactions. An example is shown in Figure 1, which illustrates the KSTAR tokamak in Daejon, Korea [1]. Introductory descriptions of tokamaks and associated plasma control problems are provided in [2], [3].

A cross-section of the KSTAR device is shown in Figure 2, with a plasma cross-section shown in the interior. The instability we consider is one in which the toroidal plasma moves either up or down in the vacuum chamber until it meets the interior vessel wall and is extinguished. One or more of the control coils is typically connected in feedback with a measurement of the vertical position to provide stabilizing control. Currents induced in control coils and passive conductors by the plasma motion provide damping, but cannot actually stabilize the instability. In KSTAR, the active control coils 1 through

J. William Helton (Fellow IEEE) is with the Faculty of Mathematics, University of California at San Diego, 9500 Gilman Drive, La Jolla, CA 92093 helton@math. ucsd. edu

Kevin J. McGown is with the Department of Mathematics, University of California at San Diego, 9500 Gilman Drive, La Jolla, CA 92093 kmcgownemath. ucsd. edu

M. L. Walker (Member IEEE) is with General Atomics, San Diego, CA 92121 walkerafusion.gat.com
14 outside of the vacuum vessel are superconducting and are used to establish the plasma equilibrium. The internal coils 15 through 18 are copper, with coils 15 and 17 dedicated to vertical position (stability) control and coils 16 and 18 used for radial position control.

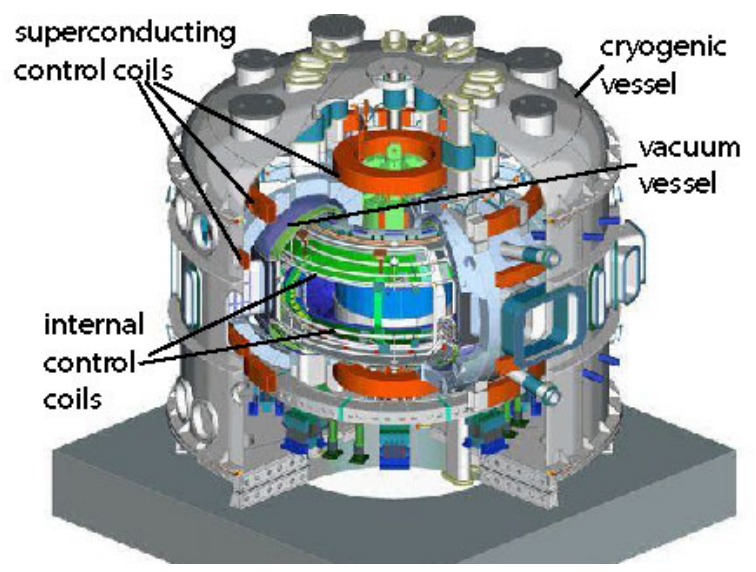

Fig. 1. Three-dimensional view illustration of the KSTAR Tokamak.

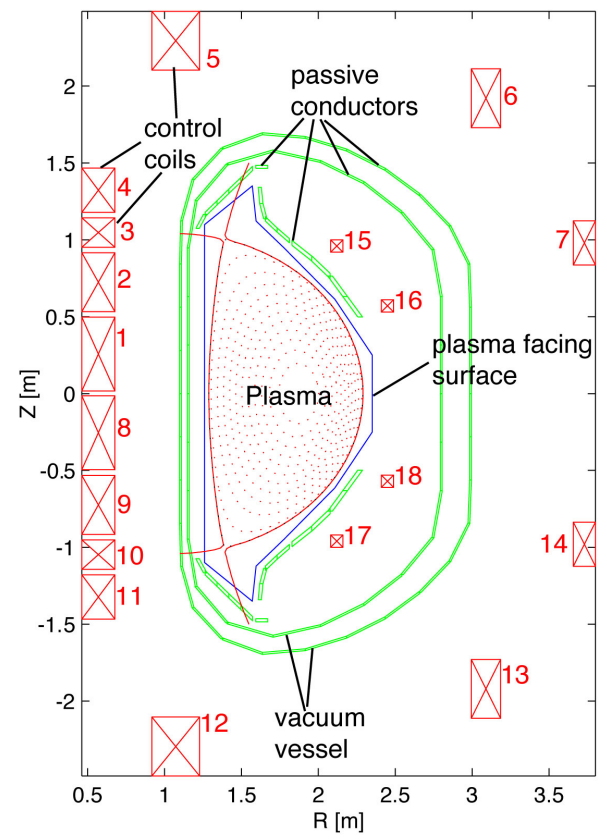

Fig. 2. Cross-section of the KSTAR Tokamak.

Feedback controllers for stabilizing the vertical instability in operating tokamaks are almost always designed based on a massless model of the plasma. However, the plasma does in fact have a small positive mass, and of all the controllers $C$ which stabilize the mass zero plasma, some stabilize the $m>0$ plasma (at least for small $m>0$ ) and others do not. 
As we shall see this is a bifurcation-like phenomenon, with our goal being to not have $C$ on a $m>0$ destabilizing branch. We provide inequalities saying exactly when this does or does not happen (see $\S I-C$ ). An illustration is Example 1.1 with a PD controller, for which choosing an $m>0$ stabilizing controller amounts to its gains having the correct sign.

Our results here work under general hypotheses which apply to a far broader class than the PD controllers currently found in today's tokamaks. Thus they resolve to a significant extent the massless vs. massive model issue when future tokamaks routinely deploy more sophisticated control algorithms (e.g. LQG or $\left.H^{\infty}\right)$. For example, confirmation of the correct inequality required for stabilizing gains (i.e. with correct sign) using physics intuition, as is presently done for PD controllers, is much more difficult in high order multivariable controllers.

We emphasize that the primary purpose of this work is not to perform a conventional robustness analysis of a particular form of controller. Rather, the primary purpose is to define conditions under which the zero-mass model can be used at all to develop controllers for stabilizing the vertical instability of the physical plasma-in-tokamak system. These conditions are of inherent theoretical interest, and also have some practical implications for plasma control design.

\section{A. Background on the Model}

The dynamic description of the plant comprising a tokamak confining an assumed axisymmetric plasma is constructed from the basic electromagnetic equation [4]

$$
M \dot{\delta I}+R \delta I+\Psi_{z} \dot{z}_{C}+\Psi_{r} \dot{r}_{C}=U \delta V
$$

where $M$ and $R$ are the mutual inductance and resistance of the toroidal conductors whose currents define the states of (1), and $\Psi_{z}, \Psi_{r}$ represent the partial derivatives of flux values at those conductors with respect to vertical $\left(z_{C}\right)$ and radial $\left(r_{C}\right)$ motion of the plasma current centroid ("center of mass" of the distributed plasma current). Toroidal currents in (respectively, voltages on) conductors are represented by the vector $I$ (resp., $V$ ) while $\delta I=I-I_{\text {eq }}$ (resp., $\delta V=V-V_{\text {eq }}$ ) represents a perturbation of the currents (voltages) from their values defining a nominal plasma equilibrium. The vector $I$ includes both currents in active control coils and in toroidal conducting vessel elements. In the following, we use the notation $\delta I=\left[\begin{array}{ll}\delta I_{c} & \delta I_{v}\end{array}\right]^{T}$ to represent a partitioning of the current vector into the $n_{c}$ active control coils and the $n_{v}$ passive (vacuum vessel) currents, $U=\left[\begin{array}{ll}\mathbf{I}_{n_{c}} & \mathbf{0}_{n_{c} \times n_{v}}\end{array}\right]^{T}$, where $\mathbf{I}_{n_{c}}$ and $\mathbf{0}_{n_{c} \times n_{v}}$ are identity and zero matrices respectively.

The motion of the current centroid for a plasma having mass $m$ can be represented by the inertial momentum equations

$$
\begin{gathered}
m \ddot{z}_{C}=f_{z} \delta z_{C}+f_{I} \delta I \\
m \ddot{r}_{C}=f_{r} \delta r_{C}+\left(\partial F_{r} / \partial I\right) \delta I
\end{gathered}
$$

where $\delta z_{C}=z_{C}-z_{C \text {,eq }}, \delta r_{C}=r_{C}-r_{C \text {,eq }}$ represent perturbed values of plasma current centroid vertical and radial coordinates relative to their values at the nominal plasma equilibrium, $f_{z}=\partial F_{z} / \partial z_{C}, f_{I}=\partial F_{z} / \partial I, f_{r}=\partial F_{r} / \partial r_{C}$, and $F_{z}$, $F_{r}$ are the total vertical and radial forces on the plasma, all quantities derived from a linearization of the plasma response around the chosen nominal plasma equilibrium. We note that $\Psi_{z}=f_{I}^{T}$ [5] [6]. The mass $m>0$, which is difficult to accurately estimate, will vary slowly relative to the dynamics of the vertical stability, and therefore may be considered as (an unknown) constant in the analysis presented here.

The equations (1) through (3) can be combined to form the overall plant model. From equation (1) we obtain

$$
M_{\#} \dot{\delta I}+R \delta I+\Psi_{z} \dot{z}_{C}=U \delta V
$$

where $M_{\#}=M+\Psi_{r}\left(\partial r_{C} / \partial I\right)$ and $\partial r_{C} / \partial I$ is computed from (3) after setting $m=0$. (Justification for setting $m=0$ for radial response but not for vertical response is discussed below.) Defining the variables $v_{z}=\dot{z}_{C}=d\left(\delta z_{C}\right) / d t, x_{z}=$ $\left[\begin{array}{ll}v_{z}^{T} & \delta z_{C}^{T}\end{array}\right]^{T}$, we can write (2) as

$$
\left(\begin{array}{cc}
0 & 1 \\
m & 0
\end{array}\right) \dot{x}_{z}+\left(\begin{array}{cc}
-1 & 0 \\
0 & -f_{z}
\end{array}\right) x_{z}+\left(\begin{array}{c}
0 \\
-f_{I}
\end{array}\right) \delta I=0
$$

Combining with (4), we obtain the matrix equation

$$
\begin{gathered}
\tilde{M} \dot{x}+\tilde{R} x=\tilde{U} \delta V, \\
x=\left(\begin{array}{c}
v_{z} \\
\delta z_{C} \\
\delta I
\end{array}\right), \tilde{M}=\left(\begin{array}{ccc}
0 & 1 & 0 \\
m & 0 & 0 \\
0 & \Psi_{z} & M_{\#}
\end{array}\right), \\
\tilde{R}=\left(\begin{array}{ccc}
-1 & 0 & 0 \\
0 & -f_{z} & -f_{I} \\
0 & 0 & R
\end{array}\right), \tilde{U}=\left(\begin{array}{c}
0 \\
0 \\
U
\end{array}\right) .
\end{gathered}
$$

If the equilibrium plasma boundary is sufficiently vertically elongated, so that $f_{z}>0$, it can be shown [5] that the system (5) possesses a single positive real eigenvalue. The eigenvector corresponding to the unstable root corresponds to a nearly rigid vertical motion of the plasma current distribution, hence the name. Stabilization of the vertical instability requires a feedback control loop that produces radial magnetic field across the plasma in response to changes in some measure of the plasma vertical position, typically the plasma current centroid position $z_{C}$ [7]. The vertical control portion of a tokamak shape and stability feedback system often takes the (PD) form

$$
\delta V(t)=-G_{p}\left(z_{C}(t)-z_{C, \mathrm{ref}}(t)\right)-G_{d} \frac{d z_{C}(t)}{d t},
$$

where $\delta V$ is the additional (nonequilibrium) voltage applied to the control coils, $z_{C}-z_{C \text {,ref }}$ is the displacement of $z_{C}$ from some reference position $z_{C \text {,ref }}$, and $d z_{C} / d t$ is the vertical velocity of the plasma. The gains $G_{p}$ and $G_{d}$ are vectors which map the scalar errors to the set of active control coils. Under many conditions, this feedback can completely stabilize the vertical instability [7].

\section{B. The Issue of Mass in Plasma Models}

For convenience of design, the mass of the plasma is neglected in virtually every design of stabilizing controllers for the vertical instability [5], [8], [6], [7], [9]. The argument that seems to be made most frequently for this approximation is that the plasma mass is only relevant on a time scale much faster than that of the instability being stabilized. However, the time scale being referred to is that of the intrinsic instability 
in the absence of passive damping by induced conductor currents. While this clearly would be a valid argument if the fast dynamics were asymptotically stable (e.g. $f_{r}<0$, the justification for setting $m=0$ in (3)), the basis for this assertion when they are unstable $\left(f_{z}>0\right.$, equation (2)) is not as clear (see, e.g., [10]).

When plasma mass is neglected, the inertial momentum equation (2) is used with $m=0$ to derive the algebraic relation $\partial z_{C} / \partial I=-f_{I} / f_{z}$, which reduces by two the state dimension of (5). However, the assumption of $m=0$ can lead to erroneous conclusions and, in particular, to PD controllers that appear to be stabilizing according to the massless analysis but will not actually stabilize the physical system. Such controllers can in fact cause the closed loop system to be far more unstable than the original open-loop system [7], [5]. Following [5], we consider a very low dimensional example that captures many aspects of the problem, to illustrate the issue more clearly.

Example 1.1: Assume that there is only one toroidal conductor, a (resistive) control coil, other than the plasma present in the system. Assume that only derivative gain is applied to the vertical position error when driving this coil, i.e. $\delta V=-G_{d} \delta \dot{z}_{C}$. If $m>0$ is maintained in the model, the maximum real part of closed loop eigenvalues, $\gamma_{C L}$, as a function of the feedback gain is shown in the top frame of Figure 3, while if the mass $m$ is set equal to zero, the most unstable eigenvalue is shown in the bottom frame. In summary, the figure shows:

The model with $m=0$ predicts that all feedback gains $U_{*} G_{d}<-1$ are stabilizing. However, the model with $m>0$ shows that all of these gains are in fact extremely destabilizing (the physically correct finding).

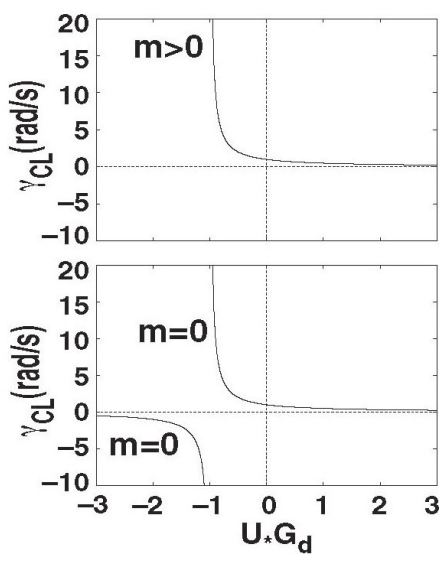

Fig. 3. Low-dimensional example.

In the figure, $U_{*}=-f_{I} /\left(f_{z} M_{*}\right)$, with $M_{*}=M_{\#}-f_{I} \Psi_{z} / f_{z}$. Details of deriving this finding are later in Example 2.5. The existence of this unphysical "stabilizing" branch is a symptom of the fact that $m=0$ is a bifurcation point of the system model. This bifurcation is due to the fact that in (2), the force $f_{z}>0$ is destabilizing, but if $m<0$, then $f_{z}>0$ becomes a stabilizing force, entirely contrary to the physics of the problem. More generally, Figure 3 shows that no pure derivative gain can stabilize the example system. In fact, [5] shows that pure derivative gain can never stabilize any plasmatokamak system in which $m>0$. It was not known a priori if the destabilizing behavior in this example has a counterpart for controllers that are more complex than PD; one objective of this work was to determine whether this is the case.

In practice, for the simple PD controller (7) and $m=0$, additional physical insight is typically used in the control design process to ensure a correctly stabilizing controller. Absent this insight or when designing a more sophisticated controller (e.g., [11], [12]) the question remains as to how to guarantee during the design process that a stabilizing controller developed with the assumption $m=0$ will also be stabilizing for the actual physical plant, since in reality $m>0$. The recent emphasis on model-based methods for design of plasma controllers, combined with uncertainty regarding the behavior of plasma models around the bifurcation point $m=0$ provided the motivation for the present work. As illustrated by Example 1.1, designing a controller using a zero mass model, then testing the controller in a simulation that also has zero mass cannot determine a priori if there will be difficulties in controlling the physical (positive mass) system. It was not known a priori if this example had a counterpart for more complex controllers. Although a small number of model-based controllers (e.g. [13], [14], [15]) have been tested experimentally, successful use of such an algorithm does not demonstrate the absence of risk, only that the particular design process used did not choose a control solution in a problematic branch of a bifurcated system.

In [5], stability properties of the open-loop system (1) were characterized and several conditions necessary for a PD controller of the form (7) to feedback-stabilize the physical (withmass) system were derived. In the present work, we address the practical problem of characterizing when a controller that has been designed using the standard but strictly incorrect zero plasma mass assumption is able to stabilize the physical system with mass $m>0$. We consider controllers whose transfer functions have the form

$$
\delta V(s)=-\left(s G_{d}+G_{p}+D(s)\right)\left(\delta z_{C}(s)-\delta z_{C, r e f}(s)\right)
$$

with $D(s)$ a vector of strictly proper rational functions, that is, $D(s) \rightarrow 0$ as $s \rightarrow \infty$. Here the length of the vector $D(s)$ equals the number of active coils. Such controllers substantially generalize the PD controller (7) to include all controllers operating on the vertical position error that can be represented by a rational function matrix with entries having relative (denominator minus numerator) degree greater than or equal to 1 .

\section{Main Results}

Our main results give necessary and sufficient conditions for such a controller to guarantee stability with (at least small) mass $m>0$ if it stabilizes the system with $m=0$. These results hold for both resistive and superconducting coils. The primary technical result (see Theorems 2.10 and 2.20) is: 
Positive Mass Test: A controller (8), which asymptotically stabilizes a massless plasma, also asymptotically stabilizes the equivalent plasma with a sufficiently small positive mass (under some minor technical conditions) if and only if the following two inequalities hold:

$$
\begin{aligned}
-f_{z}+f_{I} M_{\#}^{-1}\left[\Psi_{z}+g_{d}\right] & >0 \\
f_{I} M_{\#}^{-1} g_{p}-f_{I} M_{\#}^{-1} R M_{\#}^{-1}\left[\Psi_{z}+g_{d}\right] & <0
\end{aligned}
$$

Here the controller gain vectors $g_{p}=U G_{p}$ and $g_{d}=U G_{d}$ are defined to contain zeros in entries corresponding to the passive (vessel) conductors and equal $G_{p}$ and $G_{d}$ in entries corresponding to actively controlled coils.

If we specialize this result to $g_{p}=g_{d}=0$, we obtain the following practical consequence of this result (see Corollaries 2.12 and 2.21).

Practical Controller Test: Suppose a massless plasma is asymptotically stabilized by a strictly proper controller. Then assuming the conditions $-f_{z}+f_{I} M_{\#}^{-1} \Psi_{z} \neq 0$ and

$$
f_{I} M_{\#}^{-1} R M_{\#}^{-1} \Psi_{z}>0
$$

(automatically true for resistive coils since $f_{I}^{T}=\Psi_{z}, M_{\#}>0$ and $R>0$ ) the same plasma with sufficiently small positive mass is asymptotically stabilized by the controller if and only if

$$
f_{z}<f_{I} M_{\#}^{-1} \Psi_{z}
$$

The condition defined by the equality $f_{z}=f_{I} M_{\#}^{-1} \Psi_{z}$ is referred to by plasma physicists as the ideal stability limit; systems that exceed this limit have exponential instabilities so fast (a few microseconds) that they cannot be practically feedback stabilized. (See [7], where this limit is defined by a different calculation and considered for a low-dimensional system.) In Theorem 3 of [5], it is shown that for plasmas that are so unstable as to exceed the ideal stability limit, i.e. $f_{z}>f_{I} M_{\#}^{-1} \Psi_{z}$, the massless plasma analysis actually declares the system to be open-loop stable. The Practical Controller Test says that, for a plasma that remains below the ideal stability limit, a strictly proper feedback controller designed using the massless model will predict the closedloop behavior with a physical (positive mass) plasma. This result rigorously validates (provided the condition (12) holds) the established practice of designing a vertical stabilization controller with plasma mass assumed zero if the controller is strictly proper.

The Positive Mass Test provides caveats for controllers that are not strictly proper. Considering this test in the context of the low dimension case in Example 1.1, we see that with choice of the incorrect sign of derivative gain, it is possible to drive the system past the ideal stability limit into ideal instability (where $U_{*} G_{d}<-1$ in Figure 3), with the massless analysis predicting stability. As a side benefit, the inequality given in the Practical Controller Test also provides a method for calculating the ideal stability limit that is intuitive and easily computable from the plasma equilbrium and device geometry.

Note that power supply dynamics and control circuit filtering can be incorporated into the feedback model (8) and will frequently modify the feedback dynamics so that the overall feedback control transfer function is strictly proper. Thus, even when a nominal controller has set $G_{p}$ and $G_{d}$ to be nonzero, the asymptotic expansion which includes these external dynamics in (8) can have $g_{p}=g_{d}=0$.

\section{Derivation of Main Results}

In this section we start by describing precisely the mathematical problem corresponding to the mass zero versus nonzero issue. Then we state our main results in full detail.

In the following, we shall consider separately the case in which the diagonal matrix $R$ is positive definite, corresponding to all coils resistive, and the case in which $R$ is only positive semi-definite, corresponding to some number of control coils being superconducting.

In $\S \mathrm{II}-\mathrm{B}$ we give the solution to Problem 2.6, and in $\S \mathrm{II}-\mathrm{C}$ we translate this into the language of the tokamak and plasma system, thus giving the solution to Problem 2.1. In $\S I I-D$ we treat the superconducting case, and in $\S I I-E$ we outline a practical method for determining the maximum plasma mass for which the zero-mass feedback controller is stabilizing.

\section{A. Mathematical Problem}

In $\S$ I-A and $\S \mathrm{I}-\mathrm{B}$, we provided a description of the physical system and control problem that we wish to solve. Here (in $\S I I-A)$, we transform this description into an equivalent mathematical problem, which is then solved in $§ I I$.

We first analyze, in the frequency domain, the plasma with mass model (5) with PD feedback (7) as in [5]. First Laplace transform the matrix differential equation (5) and insert the PD feedback equation (8) (where, for the moment, we assume $d(s)=0$ ), to obtain a set of state evolution equations defined by the matrix coefficient

$$
s\left(\begin{array}{ccc}
0 & 1 & 0 \\
m & 0 & 0 \\
0 & \Psi_{z} & M_{\#}
\end{array}\right)+\left(\begin{array}{ccc}
-1 & 0 & 0 \\
0 & -f_{z} & -f_{I} \\
0 & 0 & R
\end{array}\right)+\left(\begin{array}{ccc}
0 & 0 & 0 \\
0 & 0 & 0 \\
0 & s g_{d}+g_{p} & 0
\end{array}\right)
$$

multiplying the state $x$ on the left hand side of the equality, and all terms on the right hand side representing only operations on the input reference signal.

Adding all these terms together, replacing the Laplace variable $s$ with $\lambda$, and taking the determinant of the resulting matrix, one obtains

$$
\operatorname{det}\left(\begin{array}{ccc}
-1 & \lambda & 0 \\
\lambda m & -f_{z} & -f_{I} \\
0 & \lambda \Psi_{z}+\lambda g_{d}+g_{p} & \lambda M_{\#}+R
\end{array}\right) .
$$

Clearly, the Laplace transformed system has poles where this "characteristic polynomial" (see [5]) vanishes. Thus the closed loop system is asymptotically stable if and only if this determinant is nonzero for all values of $\lambda$ in the closed RHP. Using simple row and column operations, it can be shown that this determinant is the same as the determinant (except for the sign) of the smaller matrix

$$
\tilde{G}(\lambda)=\left(\begin{array}{cc}
\lambda^{2} m-f_{z} & -f_{I} \\
\lambda \Psi_{z}+\lambda g_{d}+g_{p} & \lambda M_{\#}+R
\end{array}\right) .
$$


It follows that the closed loop system is asymptotically stable if and only if $\operatorname{det} \tilde{G}(\lambda) \neq 0$, or equivalently, the matrix $\tilde{G}(\lambda)$ is invertible, for all $\lambda$ in the closed RHP.

At this point we note that for all resistive coils both $M_{\#}$ and $R$ are positive definite and hence $\left[\lambda M_{\#}+R\right]$ is invertible for all $\lambda$ in the closed RHP [5]. Later (in $\S$ II-D) we will treat the superconductor case where it will only be assumed that $R$ is positive semidefinite instead of positive definite. In order to study more general controllers, we let $d(\lambda)=U D(\lambda)$ where $D(\lambda)$ is a column vector of rational functions such that $D(\lambda)$ is real on the real axis and $D(\lambda) \rightarrow 0$ as $\lambda \rightarrow \infty$, and consider the slightly more general matrix

$$
G(\lambda)=\left(\begin{array}{cc}
\lambda^{2} m-f_{z} & -f_{I} \\
\lambda \Psi_{z}+\lambda g_{d}+g_{p}+d(\lambda) & \lambda M_{\#}+R
\end{array}\right)
$$

We would like to answer the following:

Problem 2.1: Suppose one has chosen a controller $\left(g_{d}, g_{p}, d(\lambda)\right)$ so that the system is asymptotically stable for mass $m=0$. What are necessary and sufficient conditions so that the system will be asymptotically stable for all masses in some interval containing zero? Furthermore, we would like a method for computing the maximum allowable mass.

It is clear that the physical Problem 2.1 is equivalent to invertibility of $G(\lambda)$ for $\lambda$ in the closed RHP. Next we will express this condition in terms of another function of $\lambda$.

Definition 2.2: We define a function

$S_{m}(\lambda):=\lambda^{2} m-f_{z}+f_{I}\left[\lambda M_{\#}+R\right]^{-1}\left[\lambda \Psi_{z}+\lambda g_{d}+g_{p}+d(\lambda)\right]$.

The reason for defining this function is the following:

Remark 2.3: For any complex number $\lambda$ where $\left[\lambda M_{\#}+R\right]$ is invertible, we have

$$
G(\lambda) \text { is invertible } \Longleftrightarrow S_{m}(\lambda) \neq 0 .
$$

Proof. This is immediate since $S_{m}(\lambda)$ is just the Schur complement of the matrix $G(\lambda)$. See, for example, page 21 of [16].

In addition, this function has the following useful properties:

Lemma 2.4: For all $m \geq 0$,

1) $S_{m}(\lambda)$ is a rational function of $\lambda$,

2) $S_{m}(\lambda)=S_{0}(\lambda)+\lambda^{2} m$,

3) If $M_{\#}$ is invertible, then $S_{0}(\lambda)$ is analytic and real valued at $\infty$. In particular,

$$
S_{0}(\infty)=-f_{z}+f_{I} M_{\#}^{-1}\left[\Psi_{z}+g_{d}\right] .
$$

Proof. From the definition of $S_{m}(\lambda)$, the first and second claim are obviously true. It remains to prove the last claim. We have

$$
S_{0}(\lambda)=-f_{z}+f_{I}\left[\lambda M_{\#}+R\right]^{-1}\left[\lambda \Psi_{z}+\lambda g_{d}+g_{p}+d(\lambda)\right] .
$$

Observe that for any nonzero $\lambda$ where $\left[\lambda M_{\#}+R\right]$ is invertible we have

$$
\begin{aligned}
& {\left[\lambda M_{\#}+R\right]^{-1}\left[\lambda \Psi_{z}+\lambda g_{d}+g_{p}+d(\lambda)\right]} \\
& =\lambda^{-1}\left[M_{\#}+\lambda^{-1} R\right]^{-1} \lambda\left[\Psi_{z}+g_{d}+\lambda^{-1} g_{p}+\lambda^{-1} d(\lambda)\right] \\
& =\left[M_{\#}+\lambda^{-1} R\right]^{-1}\left[\Psi_{z}+g_{d}+\lambda^{-1} g_{p}+\lambda^{-1} d(\lambda)\right] .
\end{aligned}
$$

As $\lambda \rightarrow \infty$ we have

$$
\left[\Psi_{z}+g_{d}+\lambda^{-1} g_{p}+\lambda^{-1} d(\lambda)\right] \rightarrow\left[\Psi_{z}+g_{d}\right]
$$

and since $M_{\#}$ is invertible,

$$
\left[M_{\#}+\lambda^{-1} R\right]^{-1} \rightarrow M_{\#}^{-1} .
$$

The result follows.

Example 2.5: We illustrate the formulas above by using them to derive analytically the assertions of Example 1.1. Note that the vector of gains $g_{d}$ in this example is the scalar $G_{d}$ and recall $M_{*}=M_{\#}-f_{I} \Psi_{z} / f_{z}$, which is negative for an open-loop unstable plasma. Instability or marginal stability of the closed loop system is equivalent to $S_{m}(\lambda)=0$ for some $\lambda$ in the closed RHP (by Remark 2.3 and the italicized sentence following (14)). Multiplying $S_{m}(\lambda)$ by the scalar $\left(\lambda M_{\#}+R\right)$ and dividing by $f_{z} M_{*}$, we find that $S_{m}(\lambda)=0$ is equivalent to

$$
0=\lambda+\frac{R}{M_{*}}+\lambda U_{*} G_{d}-\lambda^{2} \frac{m\left(\lambda M_{\#}+R\right)}{f_{z} M_{*}},
$$

for all $m \geq 0$. From this equation, it is clear that the unstable eigenvalue of the open loop system is equal or approximately equal to $-\frac{R}{M *}>0$ for very small $m \geq 0$.

A necessary condition for closed-loop asymptotic stability is that the coefficients in the polynomial (16) are all positive or all negative. ${ }^{1}$ The second and third order terms in (16) that are present when $m>0$ force the sign of all coefficients to be positive for asymptotic stability (recall that $M_{\#}>0, R>0$, and $M_{*}<0$ ). Therefore any gains satisfying $U_{*} G_{d}<0$ will not asymptotically stabilize the $m>0$ system. However, they do asymptotically stabilize the $m=0$ system, by the same arguments above. This is the conclusion claimed in Example 1.1 .

Remark 2.3 and Lemma 2.4 combine to prove that we would solve Problem 2.1 if we could solve the following more general problem:

Problem 2.6: Suppose that for all $m \geq 0$,

1) $s_{m}(\lambda)$ is a rational function of $\lambda$,

2) $s_{m}(\lambda)=s_{0}(\lambda)+\lambda^{2} m$,

3) $s_{0}(\lambda)$ is analytic and real valued at $\infty$.

Suppose $s_{0}(\lambda)$ has no zeros in the closed RHP. What are necessary and sufficient conditions so that there exists $m_{*}>0$ with the property that $s_{m}(\lambda)$ has no $\lambda$ zeroes in the closed RHP for all $m \in\left[0, m_{*}\right)$. Furthermore, we would like a method for computing the maximum choice for $m_{*}$.

Since it is merely a question concerning rational functions, Problem 2.6 constitutes a considerable abstraction of Problem 2.1. For this reason, we write $s_{m}(\lambda)$ to denote a general rational function with the desired properties (as in the statement of Problem 2.6), and write $S_{m}(\lambda)$ to mean the specific function (which has these properties) given in Definition 2.2.

In this paper we will solve Problem 2.6, thereby solving Problem 2.1.

\footnotetext{
${ }^{1}$ For this second order polynomial, coefficients having a single sign is actually necessary and sufficient, but the condition is necessary for arbitrary order polynomials.
} 


\section{B. Solution in Terms of Rational Functions}

The solution to Problem 2.6 is given by the following theorem, whose proof we postpone until $\S I I I$.

Theorem 2.7: Suppose that for $m \geq 0$,

1) $s_{m}(\lambda)$ is a rational function of $\lambda$,

2) $s_{m}(\lambda)=s_{0}(\lambda)+\lambda^{2} m$,

3) $s_{0}(\lambda)$ is analytic and real valued at $\infty$.

Define a constant $c$ by writing

$s_{0}(\lambda)=s_{0}(\infty)+c \lambda^{-1}+b(\lambda)$, with $\lambda b(\lambda) \rightarrow 0$ as $\lambda \rightarrow \infty$.

Suppose that $s_{0}(\infty) \neq 0$ and $c \neq 0$. Further, suppose that $s_{0}(\lambda)$ has no zeros in the closed RHP. Then there exists $m_{*}>$ 0 such that $s_{m}(\lambda)$ has no zeros in the closed RHP for all $m \in\left[0, m_{*}\right)$ if and only if $s_{0}(\infty)>0$ and $c<0$.

It remains to give a method for finding the largest possible choice of $m_{*}$. The following definition will help us define such a method.

Definition 2.8: Let $s_{0}(\lambda)$ be a rational function. We call $\omega_{0}$ a critical value if the graph of $s_{0}(i \omega)$ crosses the positive real axis at $\omega=\omega_{0}$. Clearly there are finitely many critical values.

The following proposition, whose proof we also postpone until $\S$ III, gives the expression we seek.

Proposition 2.9: In Theorem 2.7, the largest choice for $m_{*}$ is

$$
m_{*}=\min _{k=1, \ldots, \ell}\left\{\frac{s_{0}\left(i \omega_{k}\right)}{\omega_{k}^{2}}\right\},
$$

where $\omega_{1}, \ldots, \omega_{\ell}$ are the nonzero critical values. If there are no nonzero critical values, then the quantity on the righthand side of (17) is defined to be $\infty$.

\section{Vertical Stability of the Tokamak Plasma}

Here are necessary and sufficient conditions for a massless plasma analysis to predict the vertical stability of a plasma with small mass (thus solving Problem 2.1). That is, we formally state our Positive Mass Test.

Theorem 2.10: Consider the tokamak and plasma system discussed in $\S$ I. Suppose the closed loop system is asymptotically stable for mass zero. Define

$$
\begin{aligned}
\xi & =-f_{z}+f_{I} M_{\#}^{-1}\left[\Psi_{z}+g_{d}\right] \\
\eta & =f_{I} M_{\#}^{-1} g_{p}-f_{I} M_{\#}^{-1} R M_{\#}^{-1}\left[\Psi_{z}+g_{d}\right] .
\end{aligned}
$$

Suppose that $\xi \neq 0$ and $\eta \neq 0$. Then there exists $m_{*}>0$ such that the system is asymptotically stable for all $m \in\left[0, m_{*}\right)$ if and only if $\xi>0$ and $\eta<0$.

Based on a small example set (see $\S I I-E)$ and on physical intuition, we speculate that the maximum mass $m_{*}$ will always be so large as to impose no practical constraint beyond satisfaction of the basic necessary and sufficient conditions $\xi>0$ and $\eta<0$ just described.

Example 2.11: For the low dimensional example discussed in Examples 1.1 and 2.5, asymptotic stability requires that $\xi>0$, i.e. that $-M_{\#} f_{z}+f_{I}\left[\Psi_{z}+G_{d}\right]>0$. Dividing by $f_{z}>0$ and combining terms gives $-M_{*}+f_{I} G_{d} / f_{z}>0$. Dividing again by $-M_{*}>0$ gives $U_{*} G_{d}>1$, clearly contradicting the condition $U_{*} G_{d}<-1$ derived during the massless plasma analysis.

We remark that the necessity of these conditions has already been shown in [5] for a pure PD controller. If we specialize this result to $g_{p}=g_{d}=0$, we obtain the following corollary by noting that any strictly proper controller can be expressed as an asymptotic expansion $d(\lambda)$ of the form assumed in (15).

Corollary 2.12: Consider the tokamak and plasma system discussed in $\S$ I. Suppose the system with mass 0 is asymptotically stabilized by a strictly proper controller, and suppose $f_{z} \neq f_{I} M_{\#}^{-1} \Psi_{z}$. Then there exists $m_{*}>0$ such that the close-loop system is asymptotically stable for all $m \in\left[0, m_{*}\right)$ if and only if $f_{z}<f_{I} M_{\#}^{-1} \Psi_{z}$.

Proof. For a strictly proper controller, $g_{p}=g_{d}=0$. In this case, equation (9) always holds because $f_{I}^{T}=\Psi_{z}$ and both $M_{\#}$ and $R$ are positive definite.

To prove the above results we require a lemma which explicitly computes the constant $c$ as described in Theorem 2.7.

Lemma 2.13: The function $S_{0}(\lambda)$ given in Definition 2.2 has the following expansion in $1 / \lambda$ :

$$
\begin{aligned}
& S_{0}(\lambda)=-f_{z}+f_{I} M_{\#}^{-1}\left[\Psi_{z}+g_{d}\right] \\
& \quad+\frac{f_{I} M_{\#}^{-1} g_{p}-f_{I} M_{\#}^{-1} R M_{\#}^{-1}\left[\Psi_{z}+g_{d}\right]}{\lambda}+\ldots
\end{aligned}
$$

The above series converges to $S_{0}(\lambda)$ for $|\lambda|$ sufficiently large.

Proof. We set $M=M_{\#}$ and $v=\Psi_{z}+g_{d}$ for ease of notation. We recall that $d(\lambda)$ in the definition of $S_{0}(\lambda)$ is a vector of strictly proper rational functions. Since each element of $d(\lambda)$ is a meromorphic function which is analytic at infinity, we may write

$$
d(\lambda)=\frac{d_{1}}{\lambda}+\frac{d_{2}}{\lambda^{2}}+\ldots
$$

Now we have

$S_{0}(\lambda)=-f_{z}+f_{I}[\lambda M+R]^{-1}\left[\lambda v+g_{p}+\frac{d_{1}}{\lambda}+\frac{d_{2}}{\lambda^{2}}+\ldots\right]$

Observe that, for $|\lambda|$ sufficiently large,

$$
\begin{aligned}
& {[\lambda M+R]^{-1}=\left[\lambda M\left(I+(\lambda M)^{-1} R\right)\right]^{-1}} \\
& =\left[I+(\lambda M)^{-1} R\right]^{-1}(\lambda M)^{-1} \\
& =\left[I-(\lambda M)^{-1} R+\left((\lambda M)^{-1} R\right)^{2}-\ldots\right](\lambda M)^{-1} \\
& =\frac{M^{-1}}{\lambda}-\frac{M^{-1} R M^{-1}}{\lambda^{2}}+\frac{M^{-1} R M^{-1} R M^{-1}}{\lambda^{3}}-\ldots,
\end{aligned}
$$

and hence

$$
\begin{aligned}
& {[\lambda M+R]^{-1}\left[\lambda v+g_{p}+\frac{d_{1}}{\lambda}+\frac{d_{2}}{\lambda^{2}}+\ldots\right] } \\
&= {\left[\frac{M^{-1}}{\lambda}-\frac{M^{-1} R M^{-1}}{\lambda^{2}}+\frac{M^{-1} R M^{-1} R M^{-1}}{\lambda^{3}}-\ldots\right] } \\
& \cdot\left[\lambda v+g_{p}+\frac{d_{1}}{\lambda}+\frac{d_{2}}{\lambda^{2}}+\ldots\right] \\
&=M^{-1} v+\frac{M^{-1} g_{p}-M^{-1} R M^{-1} v}{\lambda} \\
&+\frac{M^{-1} R M^{-1} R M^{-1} v-M^{-1} R M^{-1} g_{p}+M^{-1} d_{1}}{\lambda^{2}}+\ldots
\end{aligned}
$$


and the result follows.

We remark in passing that the above also gives an alternate proof to part 3 of Lemma 2.4.

Proof of Theorem 2.10. We apply Theorem 2.7 (which will be proved in $\S$ III) to the function $S_{m}(\lambda)$ given in Definition 2.2 and take into account the explicit computations of $S_{0}(\infty)$ and $c$ given in Lemmas 2.4 and 2.13.

\section{Superconducting Case}

We recall from $\S$ II-A the matrix $G$ given in (15) which governs closed loop stability:

$$
G(\lambda)=\left(\begin{array}{cc}
\lambda^{2} m-f_{z} & -f_{I} \\
\lambda \Psi_{z}+\lambda g_{d}+g_{p}+d(\lambda) & \lambda M_{\#}+R
\end{array}\right)
$$

In the superconducting case, though $R$ is positive semidefinite, it is not invertible. In fact, we have

$$
R=\left(\begin{array}{cc}
0 & 0 \\
0 & R_{r}
\end{array}\right)
$$

with $R_{r}$ invertible. We also have the partitions

$$
f_{I}=\left(\begin{array}{ll}
f_{I}^{s} & f_{I}^{r}
\end{array}\right), \quad g_{p}=\left(\begin{array}{l}
g_{p}^{s} \\
g_{p}^{r}
\end{array}\right), \quad d(\lambda)=\left(\begin{array}{l}
d^{s}(\lambda) \\
d^{r}(\lambda)
\end{array}\right),
$$

where superscripts $s$ and $r$ stand for superconducting and resistive conductors, respectively. We observe that $\lambda M_{\#}+R$ is invertible for all $\lambda$ in the closed RHP except $\lambda=0$. Here we cannot hope for the system to be asymptotically stable because we will always have a problem at $\lambda=0$. This motivates the introduction of the following terminology.

Definition 2.14: We call the tokamak and plasma system almost asymptotically stable if $G(\lambda)$ is invertible for all $\lambda$ in the closed RHP except possibly $\lambda=0$.

Note that as the terminology suggests, almost asymptotically stable is a stronger condition than stable, but weaker than asymptotically stable. The condition that $G(\lambda)$ is almost asymptotically stable means that the physical system has been stabilized to the point that all closed loop eigenvalues are in the open left half plane (asymptotic stability) except for those few eigenvalues at $\lambda=0$ due to the superconducting coils. We wish to answer the following:

Problem 2.15: Suppose one has chosen a controller $\left(g_{d}, g_{p}, d(\lambda)\right)$ so that the system is almost asymptotically stable for mass $m=0$. What are necessary and sufficient conditions so that the system will be almost asymptotically stable for all masses in some interval containing zero? Furthermore, we would like a method for computing the maximum allowable mass.

Since $R$ is not invertible in this setting, we introduce the standard generalization of the inverse of a matrix.

Definition 2.16: The Moore-Penrose pseudoinverse $A^{+}$of a matrix $A$ is the unique matrix satisfying:

1) $A A^{+} A=A$ and $A^{+} A A^{+}=A^{+}$

2) $\left(A A^{+}\right)^{*}=A A^{+}$and $\left(A^{+} A\right)^{*}=A^{+} A$

It follows that if $A^{-1}$ exists, then $A^{+}=A^{-1}$, and that for any two matrices $A$ and $B$ we have $(A B)^{+}=B^{+} A^{+}$.
Definition 2.17: We define the following function

$\hat{S}_{m}(\lambda):=\lambda^{2} m-f_{z}+f_{I}\left[\lambda M_{\#}+R\right]^{+}\left[\lambda \Psi_{z}+\lambda g_{d}+g_{p}+d(\lambda)\right]$.

The preceding definition is analogous to the function $S_{m}(\lambda)$ given in Definition 2.2. In fact, for all nonzero $\lambda$ in the closed RHP, $S_{m}(\lambda)=\hat{S}_{m}(\lambda)$ since $\left[\lambda M_{\#}+R\right]^{+}=\left[\lambda M_{\#}+R\right]^{-1}$.

Remark 2.18: For any complex number $\lambda$ where $\left[\lambda M_{\#}+R\right]$ invertible, we have

$$
G(\lambda) \text { is invertible } \Longleftrightarrow \hat{S}_{m}(\lambda) \neq 0 .
$$

Proof. This is the same as the proof of Remark 2.3.

If the closed loop tokamak and plasma system with superconductors is almost asymptotically stable at mass zero, then by the preceding remark, we know $\hat{S}_{0}(\lambda) \neq 0$ for all nonzero $\lambda$ in the closed RHP. In order to apply Theorem 2.7 to this situation we will have to impose the additional hypothesis that $\hat{S}_{0}(0) \neq 0$. In order to explicitly describe this extra condition, we now evaluate $\hat{S}_{0}$ at zero.

Lemma 2.19: If $\hat{S}_{m}(\lambda)$ is the function in Definition 2.17, then

$$
\hat{S}_{0}(0)=-f_{z}+f_{I}^{r} R_{r}^{-1}\left[g_{p}^{r}+d^{r}(0)\right] .
$$

Proof. We compute

$$
\begin{aligned}
\hat{S}_{0}(0) & =-f_{z}+f_{I} R^{+}\left[g_{p}+d(0)\right] \\
& =-f_{z}+\left(\begin{array}{ll}
f_{I}^{s} & f_{I}^{r}
\end{array}\right)\left(\begin{array}{cc}
0 & 0 \\
0 & R_{r}^{-1}
\end{array}\right)\left(\begin{array}{l}
g_{p}^{s}+d^{s}(0) \\
g_{p}^{r}+d^{r}(0)
\end{array}\right) \\
& =-f_{z}+f_{I}^{r} R_{r}^{-1}\left[g_{p}^{r}+d^{r}(0)\right],
\end{aligned}
$$

which is the desired conclusion.

We apply Theorem 2.7 to obtain:

Theorem 2.20: Consider the tokamak and plasma system with superconductors discussed above. Suppose the closed loop system (6) and (8) is almost asymptotically stable for mass zero. Further, suppose that

$$
-f_{z}+f_{I}^{r} R_{r}^{-1}\left[g_{p}^{r}+d^{r}(0)\right] \neq 0
$$

Define

$$
\begin{aligned}
\xi & =-f_{z}+f_{I} M_{\#}^{-1}\left[\Psi_{z}+g_{d}\right] \\
\eta & =f_{I} M_{\#}^{-1} g_{p}-f_{I} M_{\#}^{-1} R M_{\#}^{-1}\left[\Psi_{z}+g_{d}\right] .
\end{aligned}
$$

Suppose that $\xi \neq 0$ and $\eta \neq 0$. Then there exists $m_{*}>0$ such that the closed loop system is almost asymptotically stable for all $m \in\left[0, m_{*}\right)$ if and only if $\xi>0$ and $\eta<0$. Furthermore, the multiplicity of the eigenvalue at the origin in the system with $m>0$ is the same as in the $m=0$ system.

Proof. First we verify that the functions $\hat{S}_{m}(\lambda)$ satisfy all of the hypotheses of Theorem 2.7. Since $\hat{S}_{m}(\lambda)=S_{m}(\lambda)$ for all $\lambda$ in the closed RHP except $\lambda=0$, it is clear that $\hat{S}_{m}(\lambda)$ satisfies properties (1),(2),(3) from Theorem 2.7. In fact, the computations of $S_{0}(\infty)$ and $c$ we did in Lemmas 2.4 and 2.13 , respectively, apply here as well. The assumption that the system is almost asymptotically stable at mass zero gives us that $\hat{S}_{0}(\lambda)$ is nonzero for all $\lambda$ in the closed RHP except for possibly $\lambda=0$. But we have taken $\hat{S}_{0}(0) \neq 0$ as an additional hypothesis and hence $\hat{S}_{0}(\lambda)$ is nonzero for all $\lambda$ in the closed 
RHP. Therefore the functions $\hat{S}_{m}(\lambda)$ satisfy all the hypotheses of Theorem 2.7.

Since the situation here is a little more subtle, we prove each implication of the theorem separately. First suppose that $\xi>0$ and $\eta<0$. Theorem 2.7 tells us that there exists $m_{*}>0$ such that $\hat{S}_{m}(\lambda)$ has no zeros in the closed RHP for all $m \in$ $\left(0, m_{*}\right)$, which implies the system is almost asymptotically stable by Remark 2.18 .

Now we treat the converse. By way of contradiction suppose there exists $m_{*}>0$ such that the system is almost asymptotically stable for all $m \in\left(0, m_{*}\right)$. This implies that $\hat{S}_{m}(\lambda)$ is nonzero for all $\lambda$ in the open RHP. If $\xi>0$ and $\eta>0$, then Corollary 3.3 says that for $m$ small enough the function $\hat{S}_{m}(\lambda)$ always has a zero in the open RHP. If $\xi<0$, then Proposition 3.4 says that for some $m \in\left(0, m_{*}\right)$, the function $\hat{S}_{m}(\lambda)$ has a zero in the open RHP. In either case, we have contradicted the existence of $m_{*}$.

Finally, to see that the multiplicity of the eigenvalue at the origin is the same in either system, we merely notice that the matrix $G(0)$ is independent of $m$.

We specialize the result in Theorem 2.20 to the case of strictly proper controllers to obtain the following Corollary.

Corollary 2.21: Consider the tokamak and plasma system discussed in $\S$ I. Suppose the system with mass 0 is asymptotically stabilized by a strictly proper controller, and suppose $f_{z} \neq f_{I} M_{\#}^{-1} \Psi_{z}$ and $f_{z} \neq f_{I}^{r} R_{r}^{-1} d^{r}(0)$. Then there exists $m_{*}>0$ such that the close-loop system is asymptotically stable for all $m \in\left[0, m_{*}\right)$ if and only if $f_{z}<f_{I} M_{\#}^{-1} \Psi_{z}$.

Proof. For a strictly proper controller, $g_{p}=g_{d}=0$ and inequality (9) holds by assumption.

\section{E. Outline of the Method with an Example}

Earlier in Example 1.1 we illustrated the main problem which arises. Here we outline a practical method for computing the maximum mass stabilizable using gains chosen based on the massless plasma approximation.

1) Choose a controller $\left(g_{d}, g_{p}, d(\lambda)\right)$ so that the system is asymptotically stable for mass zero (or almost asymptotically stable for the superconducting case).

2) Perform the Positive Mass Test. That is, check that the conditions given in Theorem 2.10 hold. (For the superconducting case, this means checking the conditions of Theorem 2.20.)

3) Plot the graph $\left\{S_{0}(i \omega):-\infty<\omega<\infty\right\}$ and estimate the critical values $\omega_{1}, \ldots, \omega_{\ell}$ (see definitions 2.2 and 2.8). In the superconducting case, we use $\hat{S}_{0}(\lambda)$ instead of $S_{0}(\lambda)$ (see Definition 2.16).

4) Compute the maximum allowable mass using Proposition 2.9.

As an illustration, we describe an application of this method to a model of the KSTAR Tokamak [1], shown in figures 1 and 2. We will omit the excess details. The aim here is to give the reader an idea as to how the method is carried out. To achieve vertical stability of the plasma we set the following gains:

$$
\operatorname{gd}(15)=\operatorname{gd}(17)=0 ; \operatorname{gp}(15)=-\operatorname{gp}(17)=800 ;
$$

Again, we do not layout the details of the model, so we will omit the details as to how the gains were chosen, units, etc.

Step 1: Our software verifies that these gain values stabilize the closed loop system at mass zero.

Step 2: We perform the Positive Mass Test with the data of our model. As required by Theorem 2.10, we compute the values of $\xi$ and $\eta$ :

$$
\xi=6.2 \cdot 10^{6}, \quad \eta=-208.0 \cdot 10^{6}
$$

Since $\xi>0$ and $\eta<0$, we know that the closed loop system is asymptotically stable for sufficiently small mass. (Therefore it makes sense to compute the maximum allowable mass.) Important Note: If the chosen gain values were to fail the Positive Mass Test, they would be unusable! This step is crucial.

Step 3: We obtain the plot in Figure 4 for $S_{0}(i \omega)$. The graph crosses the real axis at 4 values of $\omega$. The $\left(\omega, S_{0}(i \omega)\right)$ pairs are $(-11,2.8 e 6),(11,2.8 e 6),(0,2.5 e 7)$, and $(\infty, 6.2 e 6)$. The crossings at $\omega_{1}=-11, \omega_{2}=+11$ yield nonzero critical values, since $w_{k} \in \mathbb{R} \backslash\{0\}$ and $S_{0}\left(i \omega_{k}\right)>0$ for $k=1,2$.

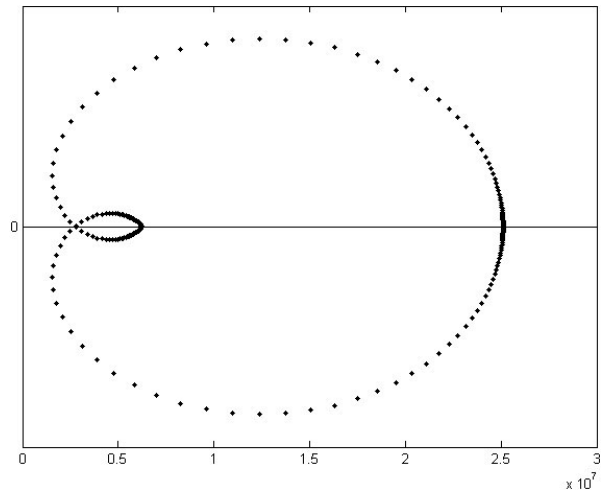

Fig. 4. Plot of $\left\{S_{0}(i \omega):-\infty<\omega<\infty\right\}$

Step 4: For $k=1,2$ we compute $S_{0}\left(i \omega_{k}\right) / \omega_{k}^{2}=2.3 \cdot 10^{4}$.

(It was expected that these two values would be equal since $\omega_{1}=-\omega_{2}$. In general, the nonzero critical values will come in pairs and the above computation only needs to be performed once for each pair.) The maximum allowable mass $m_{*}$ (in $\mathrm{kg}$ ) is the minimum of the values we just computed (see Proposition 2.9). In this simple example there is only one value; indeed, we conclude that the maximum mass which can be handled by this controller is $m_{*}=2.3 \cdot 10^{4} \mathrm{~kg}$. This is a quantity far greater than the plasma masses occurring in the tokamak, which are on the order of a milligram. This leads us to speculate that in practical situations Step 3 and Step 4 will not be important, while Step 2 of course is very important.

\section{PROOFS}

In this section we provide the proofs of the results stated in $\S$ II-B. Together, $\S$ III-A, $\S$ III-B, and $\S$ III-C make up the proof of Theorem 2.7, which is the meat of the situation and underlies all our results. Then in $§$ III-D, we give the proof of Proposition 2.9. Throughout this section we will use basic results from complex function theory. One reference, among many possible references, is [17]. 


\section{A. Counting the zeros of $s_{m}(\lambda)$}

We want to understand the behavior of the zeros of $s_{m}(\lambda)$ as we vary the parameter $m$. We start with a lemma.

Lemma 3.1: Suppose that for $m \geq 0$,

1) $s_{m}(\lambda)$ is a rational function of $\lambda$,

2) $s_{m}(\lambda)=s_{0}(\lambda)+\lambda^{2} m$,

3) $s_{0}(\lambda)$ is analytic and real valued at $\infty$.

Suppose $s_{0}(\infty) \neq 0$. Then we have the following:

1) If $s_{0}(\lambda)$ has $k$ zeros counting multiplicity, then $s_{m}(\lambda)$ has $k+2$ zeros counting multiplicity for all $m>0$. Moreover, all of these zeros move continuously in $m$.

2) We may write the zeros of $s_{0}(\lambda)$ as $\left\{\alpha_{0}^{1}, \ldots, \alpha_{0}^{k}\right\}$ and for fixed $m>0$ the zeros of $s_{m}(\lambda)$ as $\left\{\alpha_{m}^{1}, \ldots, \alpha_{m}^{k}, \beta_{m}^{1}, \beta_{m}^{2}\right\}$ so that $m \mapsto \alpha_{m}^{i}$ gives a continuous function $[0, \infty) \rightarrow \mathbb{C}$ for $i=1, \ldots, k$, and $m \mapsto \beta_{m}^{i}$ gives a continuous function $(0, \infty) \rightarrow \mathbb{C}$ for $i=1,2$. We call $\beta_{m}^{1}$ and $\beta_{m}^{2}$ the excess zeros.

3) As $m \rightarrow 0$ we have $\beta_{m}^{i} \rightarrow \infty$ for $i=1,2$.

Proof. Since $s_{0}$ is rational with $s_{0}(\infty) \neq 0$, we can write $s_{0}=p / q$ for coprime polynomials of equal degree; hence

$$
s_{m}(\lambda)=\frac{p(\lambda)+\lambda^{2} m q(\lambda)}{q(\lambda)} .
$$

When $m>0$, the numerator of (18) is a polynomial of degree $k+2$; furthermore, (18) is in lowest terms since a common zero of $p(\lambda)+\lambda^{2} m q(\lambda)$ and $q(\lambda)$ would be a zero of $p(\lambda)$. It follows that $s_{m}(\lambda)$ has $k+2$ zeros counting multiplicity.

It is straightforward to show that the zeros move continuously in $m$. This proves Part (1) of the lemma and Part (2) follows immediately from it.

It remains to show that $\beta_{m}^{i} \rightarrow \infty$ as $m \rightarrow 0$. Let $\beta_{m}$ denote either of the $\beta_{m}^{i}$. If it is not the case that $\beta_{m} \rightarrow \infty$ as $m \rightarrow 0$, then there is a sequence of nonzero real numbers $m_{n} \rightarrow 0$ such that $\beta_{m_{n}}$ converges to a complex number $\beta_{0}$, which must be a zero of $s_{0}(\lambda)$; that is, $\beta_{0}=\alpha_{0}^{j}$ for some $j$. We seek a contradiction. Setting $m=0$ and letting $\varepsilon>0$ be sufficiently small as in the above argument (where now we have $r=k$ and $\gamma_{m}^{i}=\alpha_{0}^{i}$ ) we can choose $\delta>0$ so that $N\left(\alpha_{0}^{i} ; \hat{m}\right)=N\left(\alpha_{0}^{i} ; 0\right)$ when $0 \leq \hat{m}<\delta$. By part (1) of the lemma, the total number of zeros of $s_{0}(\lambda)$ is equal to $k$. Hence for $0 \leq \hat{m}<\delta$, we have

$$
\sum^{\prime} N\left(\alpha_{0}^{i} ; \hat{m}\right)=\sum^{\prime} N\left(\alpha_{0}^{i} ; 0\right)=k
$$

where the sum is taken over a set of representatives for the collection of distinct $\varepsilon$-balls. However, if we choose $N$ large enough so that $\left|m_{N}\right|<\delta, \quad\left|\beta_{m_{N}}-\alpha_{0}^{j}\right|<\varepsilon$, then we find that

$$
\alpha_{m_{N}}^{1}, \ldots, \alpha_{m_{N}}^{k}, \beta_{m_{N}} \in \bigcup_{i=1}^{k} B_{\varepsilon}\left(\alpha_{0}^{i}\right)
$$

are all zeros of $s_{m}(\lambda)$, and hence the value of $\sum^{\prime} N\left(\alpha_{0}^{i} ; \hat{m}\right)$ is at least $k+1$, a contradiction.

\section{B. The asymptotics of the excess zeros}

If we assume that $\alpha_{0}^{1}, \ldots, \alpha_{0}^{k}$ lie in the open LHP, then for small $m>0$, we know that $\alpha_{m}^{1}, \ldots, \alpha_{m}^{k}$ also lie in the open LHP. We want to know which half-plane the $\beta_{m}^{i}$ lie in when $m>0$ is small.

Proposition 3.2: Suppose that for $m \geq 0$,

1) $s_{m}(\lambda)$ is a rational function of $\lambda$,

2) $s_{m}(\lambda)=s_{0}(\lambda)+\lambda^{2} m$,

3) $s_{0}(\lambda)$ is analytic and real valued at $\infty$.

Define a constant $c$ by writing $s_{0}(\lambda)=s_{0}(\infty)+c \lambda^{-1}+b(\lambda)$, with $\lambda b(\lambda) \rightarrow 0$ as $\lambda \rightarrow \infty$. Let $\beta_{m}$ denote either of the excess zeros (as in Lemma 3.1). If $s_{0}(\infty)>0$, then as $m \rightarrow 0$ we have

$$
\Re\left(\beta_{m}\right) \rightarrow \frac{c}{2 s_{0}(\infty)}, \quad\left|\Im\left(\beta_{m}\right)\right| \rightarrow \infty .
$$

Here, $\Re(z)$ and $\Im(z)$ denote the real and imaginary parts of a complex number $z$, respectively.

Proof. We write $s_{m}(\lambda)=s_{0}(\infty)+c \lambda^{-1}+b(\lambda)+\lambda^{2} m$ with $\lambda b(\lambda) \rightarrow 0$ as $\lambda \rightarrow \infty$. Let $\beta_{m}$ be either of the excess zeros. We see that $\beta_{m}$ satisfies

$$
s_{0}(\infty)+c \beta_{m}^{-1}+b\left(\beta_{m}\right)+\beta_{m}^{2} m=0
$$

and hence

$$
\beta_{m} s_{0}(\infty)+c+\beta_{m} b\left(\beta_{m}\right)+\beta_{m}^{3} m=0 .
$$

As $m \rightarrow 0$, we have $\beta_{m} \rightarrow \infty$ by Lemma 3.1 and hence $\beta_{m} b\left(\beta_{m}\right) \rightarrow 0$. Therefore, as $m \rightarrow 0$, we have

$$
\beta_{m} s_{0}(\infty)+c+\beta_{m}^{3} m \rightarrow 0 .
$$

We write $\beta_{m}$ in terms of its real and imaginary parts as $\beta_{m}=$ $r_{m}+j_{m}$; for ease of notation we will write $r=r_{m}$ and $j=j_{m}$, the latter being a pure imaginary number. Note that $\beta_{m}^{3}=r^{3}+3 r j^{2}+3 r^{2} j+j^{3}$ which has real part $r^{3}+3 r j^{2}$ and imaginary part $3 r^{2} j+j^{3}$. Now we decompose (19) into real and imaginary parts to find:

$$
\begin{aligned}
c+r\left[s_{0}(\infty)+\left(r^{2}+3 j^{2}\right) m\right] & \rightarrow 0 \\
j\left[s_{0}(\infty)+\left(3 r^{2}+j^{2}\right) m\right] & \rightarrow 0
\end{aligned}
$$

as $m \rightarrow 0$. The proof of the proposition will follow from our analysis of (20) and (21). First we establish two easily proved claims.

Claim: For $m>0$ small, $j_{m}$ is bounded away from 0 .

If this is not the case, then there exists $m_{n} \rightarrow 0$ such that $j_{m_{n}} \rightarrow 0$. For ease of notation, we will write $r=r_{m_{n}}, j=$ $j_{m_{n}}$, and $m=m_{n}$. In this case we must have $|r| \rightarrow \infty$ since $\beta_{m} \rightarrow \infty$ by Lemma 3.1; hence (20) gives

$$
s_{0}(\infty)+\left(r^{2}+3 j^{2}\right) m \rightarrow 0 .
$$

Finally, since $3 j^{2} m \rightarrow 0$ this implies $s_{0}(\infty)+r^{2} m \rightarrow 0$; that is, $r^{2} m \rightarrow-s_{0}(\infty)$. This requires $s_{0}(\infty) \leq 0$ in order to be possible, which contradicts our hypothesis that $s_{0}(\infty)>0$.

Claim: $r_{m}$ is bounded

If this is not the case, then there is a sequence $m_{n} \rightarrow 0$ such that $\left|r_{m_{n}}\right| \rightarrow \infty$. We seek a contradiction. For ease of 
notation, we will write $r=r_{m_{n}}, j=j_{m_{n}}$ and $m=m_{n}$. From Equation (20) we have $s_{0}(\infty)+\left(r^{2}+3 j^{2}\right) m \rightarrow 0$, which gives $\left(r^{2}+3 j^{2}\right) m \rightarrow-s_{0}(\infty)$, and from (21) and the fact that $|j|$ is bounded away from 0 , we have $\left(3 r^{2}+j^{2}\right) m \rightarrow-s_{0}(\infty)$. Combining these yields

$$
\left(8 j^{2}\right) m \rightarrow-2 s_{0}(\infty), \quad\left(8 r^{2}\right) m \rightarrow-2 s_{0}(\infty) .
$$

Subtracting, we obtain $\left(|j|^{2}+r^{2}\right) m \rightarrow 0$, so $r^{2} m \rightarrow 0$ which implies $s_{0}(\infty)=0$, contradicting $s_{0}(\infty)>0$. Having successfully proven the claim that $r_{m}$ is bounded, we dispense with our subsequences.

In light of the fact that $r_{m}$ is bounded, we now know that $\left|j_{m}\right| \rightarrow \infty$ since $\left|\beta_{m}\right| \rightarrow \infty$ by Lemma 3.1. It remains to show that the limit of $r_{m}$ exists and find its limit. Equation (21) gives

$$
s_{0}(\infty)+\left(3 r^{2}+j^{2}\right) m \rightarrow 0 ;
$$

since $r$ is bounded, this implies $s_{0}(\infty)+j^{2} m \rightarrow 0$, and hence $j^{2} m \rightarrow-s_{0}(\infty)$. Since $r$ is bounded, Equation (20) yields

$$
c+r\left[s_{0}(\infty)+3 j^{2} m\right] \rightarrow 0 .
$$

Further, since $j^{2} m \rightarrow-s_{0}(\infty)$, this implies $c+$ $r\left[-2 s_{0}(\infty)\right] \rightarrow 0$, and therefore $r \rightarrow c / 2 s_{0}(\infty)$. This completes the proof of the proposition.

Now we completely understand the case $s_{0}(\infty)>0$ :

Corollary 3.3: Under the hypotheses of Proposition 3.2 plus the assumption that $s_{0}(\lambda)$ has no zeros in the closed RHP, we have:

1) If $s_{0}(\infty)>0$ and $c<0$, then there exists $m_{*}>0$ such that $s_{m}(\lambda)$ has no zeros in the closed RHP for all $m \in\left(0, m_{*}\right)$.

2) If $s_{0}(\infty)>0$ and $c>0$, then there exists $m_{*}>0$ such that $s_{m}(\lambda)$ has at least one zero in the open RHP for all $m \in\left(0, m_{*}\right)$.

Proof. This follows immediately from the continuity of the zeros given by Lemma 3.1, and the asymptotics given in Proposition 3.2.

\section{C. $s_{0}(\infty)<0$ begets instability}

From the analysis given up until this point, it is not clear what happens to the $\beta_{m}^{i}$ as $m \rightarrow 0$ in the case where $s_{0}(\infty)<$ 0 ; indeed, it is not even clear whether such a limit will exist. The following proposition gives a more delicate analysis of the case where $s_{0}(\infty)<0$.

Proposition 3.4: Suppose that for $m \geq 0$,

1) $s_{m}(\lambda)$ is a rational function of $\lambda$,

2) $s_{m}(\lambda)=s_{0}(\lambda)+\lambda^{2} m$,

3) $s_{0}(\lambda)$ is analytic and real valued at $\infty$.

Suppose $s_{0}(\infty)<0$, and let $\delta>0$ be given. Then there exists $m \in(0, \delta)$ such that $s_{m}(\lambda)=0$ for some $\lambda$ in the open RHP.

Proof. For any $m \geq 0$, we have $s_{m}(\lambda)=0$ if and only if $s_{0}(\lambda)+\lambda^{2} m=0$. Making the substitution $\lambda=1 / z$ we find that this is true exactly when

$$
F_{m}(z):=m+z^{2} s_{0}(1 / z)=0
$$

for some $z \neq 0$. Since $s_{0}(1 / z)$ is analytic at $z=0$ we may write

$$
s_{0}(1 / z)=s_{0}(\infty)+z g(z)
$$

where $g(z)$ is analytic at $z=0$. By taking $\delta$ small enough, we may assume, without loss of generality, that $g(z)$ is analytic and this expression for $s_{0}(1 / z)$ holds for all $z \in B_{2 \delta}(0)$. This yields

$$
F_{m}(z)=f_{m}(z)+z^{3} g(z)
$$

where we define $f_{m}(z):=m+z^{2} s_{0}(\infty)$. Choose $\varepsilon>0$ such that

$$
\varepsilon<\delta, \quad \frac{\varepsilon^{2}\left|s_{0}(\infty)\right|}{2}<\delta
$$

and $|z| \leq \varepsilon$ implies

$$
|z g(z)|<\frac{\left|s_{0}(\infty)\right|}{2}
$$

it is possible to accomplish the latter condition (22) since $g(z)$ is analytic at $z=0$ and $\left|s_{0}(\infty)\right|>0$ by hypothesis. Fix

$$
m:=\frac{\varepsilon^{2}\left|s_{0}(\infty)\right|}{2}
$$

and observe that $m \in(0, \delta)$. Define

$$
\Omega:=\{z \in \mathbb{C}|\Re z>0,| z \mid<\varepsilon\} \text { and } z_{0}:=\sqrt{\frac{m}{\left|s_{0}(\infty)\right|}} .
$$

Since $s_{0}(\infty)<0$, we have $f_{m}\left(z_{0}\right)=0$. Furthermore,

$$
z_{0}^{2}=\frac{m}{\left|s_{0}(\infty)\right|}<\frac{2 m}{\left|s_{0}(\infty)\right|}=\varepsilon^{2},
$$

and hence $z_{0} \in(0, \varepsilon)$; that is, $z_{0} \in \Omega$. Clearly, the only other zero of $f_{m}(z)$ is $-z_{0} \notin \Omega$. Also, being a polynomial, $f_{m}(z)$ has no poles. Since $g(z)$ is analytic in $B_{2 \delta}(0)$, we know that $F_{m}(z)$ has no poles in $B_{2 \delta}(0) \supseteq \bar{\Omega}$.

We will shortly apply Rouché's Theorem (see, for example, [17]) to conclude that $F_{m}(z)$ has exactly one zero in $\Omega$. In order to satisfy the appropriate hypotheses, we must show that $\left|z^{3} g(z)\right|<\left|f_{m}(z)\right|$ for all $z \in \partial \Omega$. In particular, this will also demonstrate that $F_{m}(z)$ has no zeros on $\partial \Omega$. Since $|z| \leq \varepsilon$ for all $z \in \bar{\Omega}$, taking into account (22), it suffices to show that

$$
\frac{\left|f_{m}(z)\right|}{\left|z^{2}\right|} \geq \frac{\left|s_{0}(\infty)\right|}{2}
$$

for all $z \in \partial \Omega$. First we consider the portion of $\partial \Omega$ that lies on the imaginary axis. If $z=i \omega$ with $\omega$ real and $|z| \leq \varepsilon$, then we have

$$
\frac{\left|f_{m}(z)\right|}{\left|z^{2}\right|}=\frac{m}{\omega^{2}}+\left|s_{0}(\infty)\right|>\frac{\left|s_{0}(\infty)\right|}{2} .
$$

(Note that the above relied again on the fact that $s_{0}(\infty)<0$.) Now we treat the remaining portion of $\partial \Omega$. There $|z|=\varepsilon$ and

$$
\begin{aligned}
\frac{\left|f_{m}(z)\right|}{\left|z^{2}\right|} & =\left|\frac{m}{z^{2}}+s_{0}(\infty)\right| \\
& \geq\left|s_{0}(\infty)\right|-\frac{m}{\varepsilon^{2}}=\frac{\left|s_{0}(\infty)\right|}{2} .
\end{aligned}
$$

The last equality following from (23).

Having satisfied the necessary hypotheses, we invoke Rouché's Theorem to conclude that $F_{m}(z)$ has a zero, say 
$z_{0} \in \Omega$. It follows that $\gamma:=1 / z_{0}$ is in the open RHP and $s_{m}(\gamma)=0$.

Now the proof of Theorem 2.7 follows immediately from from Corollary 3.3 and Proposition 3.4.

\section{The largest value for $m_{*}$}

Having completed the proof of Theorem 2.7, we are now in a position to prove Proposition 2.9 . First we need a preliminary lemma.

Lemma 3.5: Suppose that for $m \geq 0$,

1) $s_{m}(\lambda)$ is a rational function of $\lambda$,

2) $s_{m}(\lambda)=s_{0}(\lambda)+\lambda^{2} m$.

If $\omega$ is a nonzero critical value (see Definition 2.8), then for $m=s_{0}(i \omega) / \omega^{2}$, the function $s_{m}(\lambda)$ has a zero on the imaginary axis.

Proof. By definition, $\omega$ being a nonzero critical value implies that $s_{0}(i \omega)$ is a positive real number and hence $m=$ $s_{0}(i \omega) / \omega^{2}$, is a positive real number with $s_{0}(i \omega)-\omega^{2} m=0$. Substituting $\lambda=i \omega$ into the equation $s_{m}(\lambda)=s_{0}(\lambda)+\lambda^{2} m$ yields $s_{m}(i \omega)=s_{0}(i \omega)-\omega^{2} m=0$. That is, $s_{m}(\lambda)$ has a zero on the imaginary axis.

Proof of Proposition 2.9. Suppose that the hypotheses of Theorem 2.7 are satisfied, and further suppose that $s_{0}(\infty)>0$ and $c<0$. In this case, Theorem 2.7 says that there exists $m_{*}>0$ so that $s_{m}(\lambda)$ has no zeros in the closed RHP for all $m \in\left[0, m_{*}\right)$. Choose the largest possible value for $m_{*}$, allowing the possibility that $m_{*}=\infty$. Now define

$$
\hat{m}:=\min _{k=\omega_{1}, \ldots, \omega_{\ell}}\left\{\frac{s_{0}\left(i \omega_{k}\right)}{\omega_{k}^{2}}\right\},
$$

where $\omega_{1}, \ldots, \omega_{\ell}$ are the critical values (see Definition 2.8), taking the convention that $\hat{m}=\infty$ if there are no critical values. We must show that $\hat{m}=m_{*}$. From Lemma 3.5 we already know $m_{*} \leq \hat{m}$. To show that $m_{*} \geq \hat{m}$, which would complete the proof, we must show that for all $m \in(0, \hat{m})$, the function $s_{m}(\lambda)$ has no zeros in the closed RHP.

By way of contradiction, suppose that there exists $m \in$ $(0, \hat{m})$ such that $s_{m}(\lambda)$ has a zero in the closed RHP. By Corollary $3.3, s_{m}(\lambda)$ has no zeros in the closed RHP when $m>0$ is sufficiently small. Moreover, Lemma 3.1 tells us that when $m \in(0, \infty)$, all of the zeros of $s_{m}(\lambda)$ move continuously in $m$. Thus there is some intermediate value of $m$ for which $s_{m}(\lambda)$ has a zero on the imaginary axis. That is, $s_{m}(i \omega)=0$ for some $\omega \in \mathbb{R}$. We note that $\omega \neq 0$, since if $\omega$ was equal to zero, we would have $s_{0}(0)=s_{m}(0)=0$, violating the hypothesis that $s_{0}(\lambda)$ has no zeros in the closed RHP. Since $s_{m}(i \omega)=s_{0}(i \omega)-\omega^{2} m$, we have $s_{0}(i \omega)=\omega^{2} m \in(0, \infty)$ and hence the graph of $s_{0}(i \omega)$ crosses the positive real axis at this particular value of $\omega$. Thus $\omega$ is a critical value and we conclude that

$$
m=\frac{s_{0}(i \omega)}{\omega^{2}} \geq \hat{m},
$$

which contradicts the assumption that $m \in(0, \hat{m})$.

An alternative approach to our analysis that has been suggested is to use methods based on Kharitonov's Theorem. These methods could determine stability of the polynomial produced by multiplying the rational function $S_{m}(\lambda)$ by its denominator. for a parameter $m$ in an interval $[0, b]$, but one must check stability of $S_{b}(\lambda)$ at the end point $b$. It is natural to apply a Routh-Hurwitz test to do this. Indeed, Humphreys and Walker have attempted this with limited success, as the resulting symbolic (i.e. non-numerical) expressions became far too complex to analyze [5].

\section{CONCLUSIONS}

In this paper, we have derived necessary and sufficient conditions for a controller of a particular form to guarantee stability with plasma mass $m>0$ if it stabilizes the tokamak plasma vertical instability with plasma mass assumed to be zero. We analyze a fundamental bifurcation that occurs at $m=$ 0 . The result of this analysis provides the rigorous foundation for the common practice of designing controllers assuming $m=0$. The class of controllers considered significantly generalizes the PD controller that is used to represent vertical stabilization algorithms on existing tokamaks, but is limited to controllers that operate on a measured plasma vertical position error signal. The conditions for $m>0$ stabilization are on the characteristic parameters of the open-loop system and on the two leading terms of the generalized controller, corresponding to the gains $G_{p}$ and $G_{d}$ of the PD controller (7).

Note that the main issue addressed in this work is not to determine how large the mass can be for the closed loop to remain stable. Rather, we determine when there exists any $m>0$ for which the closed loop is stable. If one chooses a controller with gains violating either of our Positive Mass Test inequalities (9),(10), then no plasma with positive mass is stabilizable, notwithstanding the fact that the $m=0$ closed loop is stable. The results given plus a conjecture based on physical intuition and a few examples such as the one in $\S$ II-E suggest that if the closed loop is stable for some $m>0$, then it is stable for all possible physically relevant plasma masses.

In previous analyses (e.g. [7]) of plasma vertical stabilization control, the use of pure proportional plus pure derivative gain as in equation (7) has been assumed to be representative of the main features of the problem. We have found however that this simplification is in fact not completely representative of practical feedback controllers. One obtains strikingly different conclusions about closed loop behavior depending on what one assumes about the controller's asymptotic behavior at infinity. When external control circuits and power supplies are accounted for, practical controllers tend to be strictly proper and therefore our Practical Controller Test, based on the Corollaries 2.12 and 2.21, is more relevant. These corollaries show that for equilibria that are not too unstable relative to the passive stabilization of the device, i.e., when $f_{z}<f_{I} M_{\#}^{-1} \Psi_{z}$, controllers that stabilize the massless model will also stabilize the physical $(m>0)$ plasma. Conversely, when the inequality is reversed, a massless model analysis will not suffice to predict closed-loop stability of the physical system.

Even though this paper concerns a branching type phenomenon, our results may alternatively be viewed as control robustness results, but only with respect to the uncertain plasma mass. If the two inequalities (9),(10) (see also Theorem 
2.20) are satisfied by a controller of the form given in (8) that is stabilizing for $m=0$, we conjecture that this controller robustly stabilizes for all (practical) $m>0$. Uncertainties other than plasma mass (e.g. in the coupling between plasma and conductors, plasma resistivity, plasma current distribution, pure delays, etc.) can also have an impact on the controller performance, but none of these uncertainties is as fundamental as a branching in the basic system response. Additional practical constraints can potentially lead to a less stable closed loop system than the ideal case analyzed here, so obviously a controller design might not be robust to the effects we have not analyzed.

\section{ACKNOWLEDGEMENTS}

Professor Helton was partially funded by NSF grant DMS 0700758 and from the Ford Motor Company and Kevin McGown by the same NSF grant. Mike Walker was supported by the U.S. Department of Energy under Contract DE-FC0204ER54698.

We gratefully acknowledge the National Fusion Research Institute of Korea for allowing us to use models of the KSTAR Tokamak for the practical examples.

\section{REFERENCES}

[1] Y.K.Oh, et.al., Completion of the KSTAR construction and its role as ITER pilot device, Fusion Engineering and Design, Volume 83, Issues 7-9, December 2008

[2] IEEE Control Systems Magazine, special issue on Control of Tokamak Plasmas, Oct. 2005, v.25, no.5

[3] IEEE Control Systems Magazine, special issue on Control of Tokamak Plasmas II, Apr. 2006, v.26, no.2

[4] M. L. Walker, D. A. Humphreys, Valid coordinate systems for linearized plasma shape response models in tokamaks. Fusion Science and Technology, v.50, no.4, Nov. 2006

[5] M. L. Walker and D. A. Humphreys, On Feedback Stabilization of the Tokamak Plasma Vertical Instability, Automatica, v. 45, no. 3, March 2009, 665-674

[6] Ambrosino, G., Albanese, R., Magnetic Control of Plasma Current, Position, and Shape in Tokamaks, A survey of modeling and control approaches, IEEE Cont. Syst. Mag., v.25, no.5, Oct. 2005

[7] E. A. Lazarus, J. B. Lister, G. H. Neilson, Control of the Vertical Instability in Tokamaks, Nucl. Fusion 30, 1990, 111.

[8] G. Ambrosino, M. Ariola, G. De Tommasi, A. Pironti, A. Portone, "Design of the plasma position and shape control in the ITER tokamak using in-vessel coils," IEEE Transactions on Plasma Science, vol. 37, no. 7, pp. 1324-1331, July 2009.

[9] R. Albanese, F. Villone, The Linearized CREATE-L Plasma Response Model for the Control of Current, Position and Shape in Tokamaks, Nucl. Fusion, Vol. 38, No. 5 (1998), 723

[10] K. Guemghar, B. Srinivasan, Ph. Mullhaupt, D. Bonvin, Predictive Control of Fast Unstable and Nonminimum-phase Nonlinear Systems, Proc. of American Control Conference, Anchorage, AK, 2002

[11] D.A. Ovsyannikov, E.I. Veremey, A.P. Zhabko, A.D. Ovsyannikov, I.V. Makeev, V.A. Belyakov, A.A. Kavin, M.P. Gryaznevich and G.J. McArdle, Mathematical methods of plasma vertical stabilization in modern tokamaks, Nucl. Fusion 46 (2006)

[12] Al-Husari, M.M.M.; Hendel, B.; Jaimoukha, I.M.; Kasenally, E.M.; Limebeer, D.J.N.; Portone, A., Vertical stabilisation of Tokamak plasmas, Proc. of 30th IEEE Conference on Decision and Control, 1991, 11-13 Dec 1991, pp. 1165 - 1170, vol.2

[13] P. Vyas, D. Mustafa, and A. W. Morris, Vertical Position Control on COMPASS-D, Fusion Science \& Technology, v. 33, no. 2, March 1998

[14] M. Ariola et al., "Design and experimental testing of a robust multivariable controller on a tokamak," IEEE Trans. Control Syst. Technol., vol. 10, no. 5, pp. 646-653, Sep. 2002.

[15] M.L. Walker, J.R. Ferron, D.A. Humphreys, R.D. Johnson, J.A. Leuer, B.G. Penaflor, D.A. Piglowski, M. Ariola, A. Pironti, E. Schuster, Nextgeneration plasma control in the DIII-D tokamak, Fusion Engineering and Design 66/68 (2003) 749-753
[16] R. A. Horn and C. R. Johnson, Matrix Analysis, Cambridge University Press, New York, 1985.

[17] J. B. Conway, Functions of One Complex Variable I, Springer-Verlag, New York, 1978. 